\title{
Demonstration of a plasma mirror based on a laminar flow water film.
}

\author{
Dmitriy Panasenko*, Anthony J. Shu, Anthony Gonsalves, Kei Nakamura, Nicholas \\ H. Matlis, Csaba Toth and Wim P. Leemans \\ Lawrence Berkeley National Laboratory, University of California, Berkeley, California 94720
}

\begin{abstract}
A plasma mirror based on a laminar water film with low flow speed $(0.5-2 \mathrm{~cm} / \mathrm{s})$ has been developed and characterized, for use as an ultra-high intensity optical reflector. The use of flowing water as a target surface automatically results in each laser pulse seeing a new interaction surface and avoids the need for mechanical scanning of the target surface. In addition, the breakdown of water does not produce contaminating debris that can be deleterious to vacuum chamber conditions and optics such as is the case when using conventional solid targets. The mirror exhibits $70 \%$ reflectivity, while maintaining high-quality of the reflected spot.
\end{abstract}

\section{INTRODUCTION}

The effect of self-induced plasma shuttering and subsequent reflection of laser beams from the ensuing critical density layer of the plasma is commonly referred to as plasma mirror (PM) production. Generation of a PM can occur when the early part of a ultrashort, typically sub-picosecond laser pulse is incident on a material with intensity sufficient to ionize it and produce plasma with the electron density exceeding critical $(2 \mathrm{x}$ $10^{21} \mathrm{~cm}^{-3}$ for $800 \mathrm{~nm}$ light) that reflects the remainder of the laser pulse [1]. For ultrashort, intense laser pulses, this plasma layer will have limited expansion on the femtosecond scale and can therefore act as a high quality reflective surface for the rest of the laser pulse. By appropriately selecting the interaction parameters (i.e. the intensity and the spot size), a significant portion of the laser energy can be specularly reflected.

Plasma mirrors have proven to be extremely useful for improving the temporal contrast of multi-TW, femtosecond laser systems [1-3]. Such systems frequently suffer from high levels of unwanted amplified spontaneous emission (ASE) and relatively high level pre-pulses on the sub-nanosecond timescale. For peak intensities associated with 10-100 TW laser systems, these pre-pulses can have enough intensity to generate plasma on the target before the main laser pulse arrives, therefore altering the physics of the intended experimental study. Reflecting the laser pulse off the plasma mirror created on anti-reflection coated optics that initially transmit the low intensity pre-pulses and ASE pedestal, allows significant improvement of the pulse contrast. Plasma mirrors also showed promise for high harmonic generation through coherent wake emission and oscillating relativistic mirror effects [3]. Additional applications may include steering of high intensity ultrashort pulse laser beams close to a focal point where the use of regular optics is precluded by material breakdown. Such steering may be needed in applications where the size of the optical system has to be minimized such as staging of laser plasma accelerators [4]. 
Most of the research on the development and application of plasma mirrors has been conducted using solid dielectric targets. Solid targets are attractive because they can be produced with surfaces of high quality. As the plasma is formed on a femtosecond timescale, the profile of the plasma mirror surface matches that of the unionized target. However, there are two critical disadvantages to the solid-state approach. First, mechanical scanning has to be used in order to provide a fresh target surface for every laser shot. While this is acceptable for lasers with repetition rates in the range of 1 to 10 $\mathrm{Hz}$, a significant effort is now devoted to the development of multi-TW laser systems operating at $\mathrm{kHz}$ rates [5] where mechanical scanning of the target will be much more challenging. Standard approach of translating a flat transparent optics across the focus of the laser will fast run out of available target surface limiting the possibility of continuous operation of the mirror. Second, laser-induced breakdown of the material surface that precedes the formation of the plasma mirror can produce large amounts of debris [6]. The debris can result in decreased performance and even damage of the conventional optics that are located in the vicinity of the target.

To address these issues, an alternative approach was pursued in which the target was replaced with a laminar-flow, planar liquid jet. As the surface is continuously refreshed, the target requires no replacement, making it ideal for use in multi-TW, kHz systems. The liquid jet concept was first demonstrated by Backus et al. [7] in which a nozzle developed for commercial dye lasers was used with ethylene glycol. Ethylene glycol was chosen for its high viscosity which results in liquid surfaces of good optical quality as well as for its low saturated vapor pressure that allows operation in vacuum. However, its carbon-based composition makes it undesirable from a contamination perspective.

Using water as a target would eliminate the contamination, since hydrogen and oxygen will not deposit on the optical surfaces. The development of a planar water jet however, represents a significant challenge due to the low viscosity of water (about 20 times smaller than that of ethylene glycol). Avoiding turbulence that would make the jet unusable as an optical reflection surface requires operation at low flow velocities to ensure a small Reynolds number. Reducing the flow velocity in turn makes the water jet susceptible to surface-tension induced collapse of the planar jet before the surface becomes stable.

In order to prevent this collapse a guiding structure can be used, where a liquid film flows between two guiding wires. Wire guided films have been used previously for Raman spectroscopy of water and proved to be a reliable way of creating stable laminar flow water films [8]. The quality of the specular reflection off such films, which is essential for their use as plasma mirrors, has not been characterized previously.

In this paper, a plasma mirror based on a laminar flow water film is demonstrated. The mirror reflectivity reached $70 \%$, which is similar to reflectivities obtained from solid targets. This approach will be useful for applications with future multi-TW laser systems working at high repetition rates.

\section{DESIGN OF LAMINAR FLOW PLANAR WATER JET.}

In the experiments, a water film was formed in a 4-mm diameter round opening drilled in 60-micron thick piece of stainless steel shim. Water is fed onto the shim from a 0.5 $\mathrm{mm}$ round glass nozzle positioned at 60 degrees above the opening, as shown in Fig. 1. 
The flow from the nozzle was supplied by a reservoir filled with water and driven by gravity, allowing for 1 hour of continuous operation. A straightforward modification of the apparatus would allow for recirculation and a continuous operation [7].

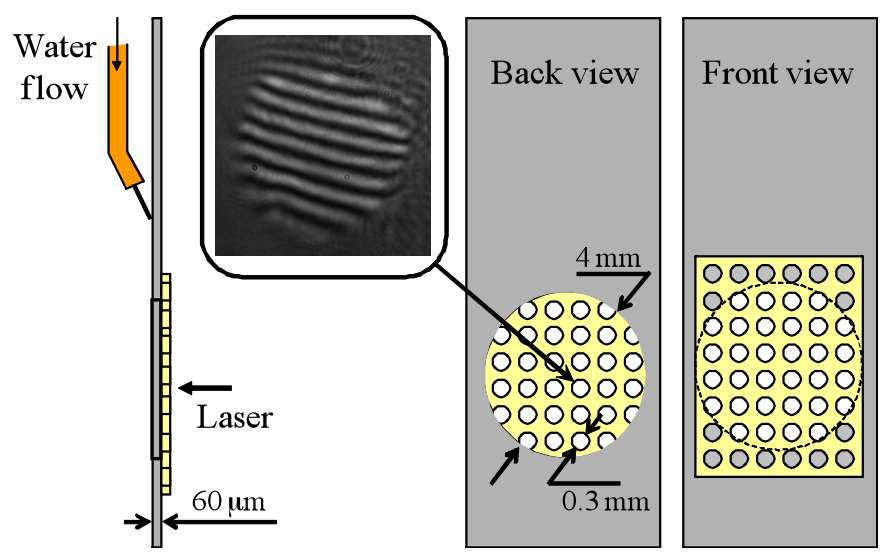

Figure 1. Design of the water jet target for plasma mirror. Inset: Fringe pattern from a single hole in the mesh used for flow stabilization

A 125 -micron thick bronze mesh with $0.35 \mathrm{~mm}$ holes was glued on the back of the shim, so that the water flowed on top of the mesh. Due to surface tension, water did not penetrate into the openings of the mesh. The latter was confirmed by observing the fringe pattern between a beam transmitted through a single hole in the mesh and a reference beam in a Michelson interferometer. The fringe pattern under standard operating conditions (in air), shown in the inset in Fig.1, is almost straight, indicating little or no curvature of the surface. When the water was forced into the mesh openings, the fringes would form a characteristic Newton ring pattern. A Ti:Sapphire laser pulse, split-off before the final compressor of a chirped pulse amplification system was used to measure the thickness of the jet using time-of-flight method in a Michelson interferometer. The guiding structure with the water film was positioned in one of the arms and the shift of the fringe pattern due to pulse propagation through the water film was analyzed. The 30$\mathrm{nm}$ bandwidth of the pulse allowed measurement with accuracy of about 15 microns. At the flow speeds between $0.5 \mathrm{~cm} / \mathrm{s}$ and $2 \mathrm{~cm} / \mathrm{s}$ the thickness of the jet was found to depend almost linearly on the velocity following the equation $d[\mu \mathrm{m}]=316 \times v[\mathrm{~cm} / \mathrm{s}]+102$. At higher velocities the thickness measurements were not repeatable and this is attributed to instabilities on the back (free) surface of the jet. At speeds lower than $0.5 \mathrm{~cm} / \mathrm{s}$, the flow out of the capillary nozzle would break into discreet drops. This range of velocities for which the thickness measurement was repeatable corresponds to Reynolds numbers between 1.5 and 16.5. Instability at such low Reynolds numbers is not surprising since it has been shown that a free falling film is unstable (i.e. a class of undamped waves exists) at all finite Reynolds numbers [9]. However the rate of amplification for the instability falls with Reynolds number which explains an observed velocity range where the flow is stable.

It should be noted that the bronze mesh was not necessary in order to achieve stable laminar flow of water under atmospheric pressure. In this case, the water film would be 
simply formed in the round opening similar to the wire-guided jet described in ref.[8]. In practice, however, the water jet plasma mirror must be operated in vacuum to avoid selffocusing of the high-power laser and laser-induced breakdown of the air upstream of the jet. It was found that under these low-ambient-pressure conditions, the water film was susceptible to rupture in the absence of the mesh. This may be due to an instability triggered by evaporation of the liquid, such as the Bernard or Marangoni instabilities [10]. The wave numbers of such instabilities, in units of inverse thickness of the layer, are usually of the order of unity, which implies that the spatial period of instability is of the order of the layer thickness [11]. The mesh, having a period of the same order, limits the free surface of the liquid and can therefore stabilize the free surface. A detailed analysis of the stability of the free surface is outside the scope of this paper.

\section{PERFORMANCE OF THE PLASMA MIRROR}

The performance of the plasma mirror was characterized by determining its reflectivity and surface quality. This was done by measuring the energy and mode shape of $40 \mathrm{fs}, 800 \mathrm{~nm}$ pulses from a Ti:Sapphire amplifier system before and after reflection as a function of incident intensity The temporal contrast of the pulse was enhanced by a crossed polarized wave (XPW) system [12]. Figure 2 shows a representative example of the full power laser contrast as used during the experiments. The measurement was made using a commercial third-order cross-correlator. The contrast with respect to the prepulse was about $10^{-5}$ while the amplified spontaneous emission background was suppressed by more than $10^{-8}$. The pedestal between $-5 \mathrm{ps}$ and $-1 \mathrm{ps}$ has been reduced in subsequent work and may be due to a misalignment of the laser path into the cross-correlator.

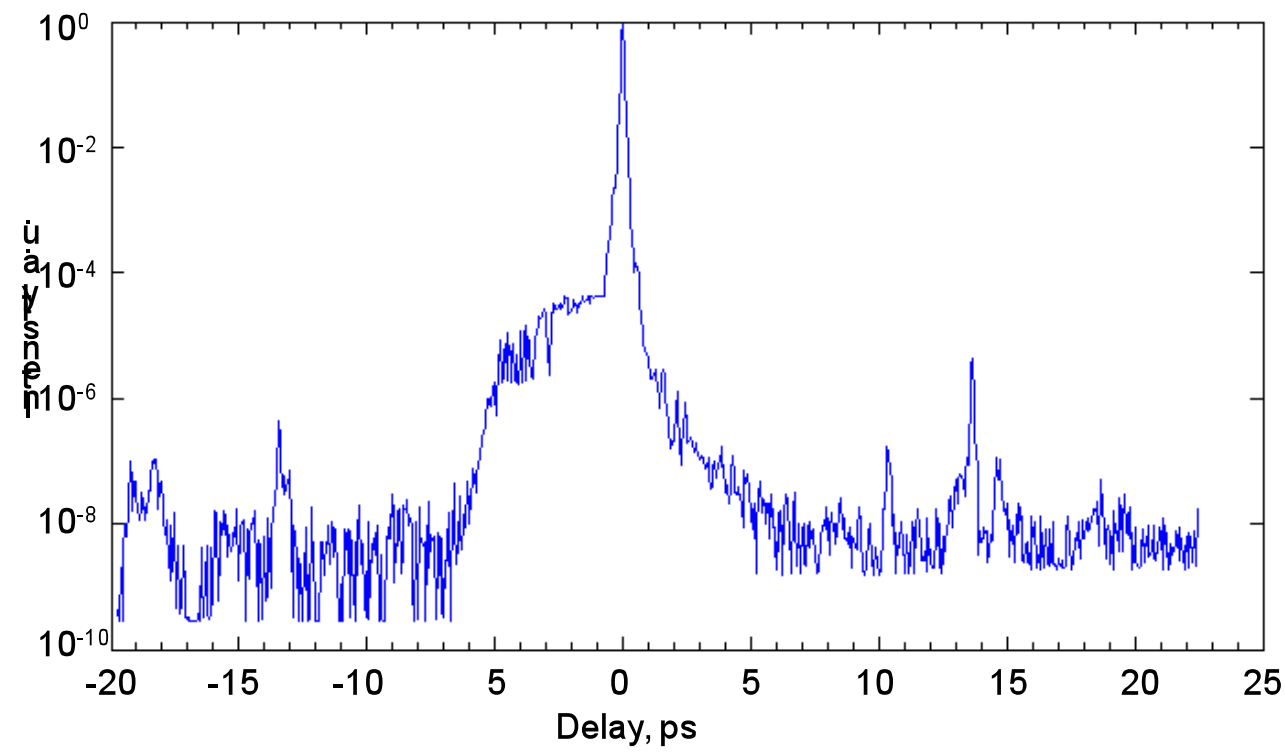

Figure 2. A representative example of a third order correlation of the laser pulse illustrating pulse contrast used during the experiments. The pedestal between $-5 \mathrm{ps}$ and $-1 \mathrm{ps}$ has been reduced in subsequent work.

For the reflectivity measurements, the water jet was mounted in a vacuum chamber. An s-polarized beam entered the chamber through an AR coated window and was 
focused on the water film, at a 45-degree incidence angle, with a $140-\mathrm{mm}$ focal distance achromatic lens positioned inside the chamber. The lens was mounted on a remotely controlled translation stage in order to adjust the position of the focus relative to the water film. Visible luminescence from the plasma at laser intensities close to the dielectric breakdown threshold was used to position the focus of the beam on the target. A conjugate pair of plano-convex $300 \mathrm{~mm}$ focus lenses outside the chamber collected reflected light and directed it to a CCD camera. The CCD camera was calibrated against the input energy of the laser. The pulse energy was detected on every shot with a photodiode sampling the $0^{\text {th }}$ order reflection from one of the compression gratings. The photodiode was calibrated against an energy meter (Coherent J-25a). The energy of the laser pulse was tuned by varying the timing of the Q-switched pulses on the Nd:YAG pump lasers, which resulted in relative time delay between the 532-nm pump pulses and the $800 \mathrm{~nm}$ signal pulse on the Ti:Sapphire crystal in the final amplifier.

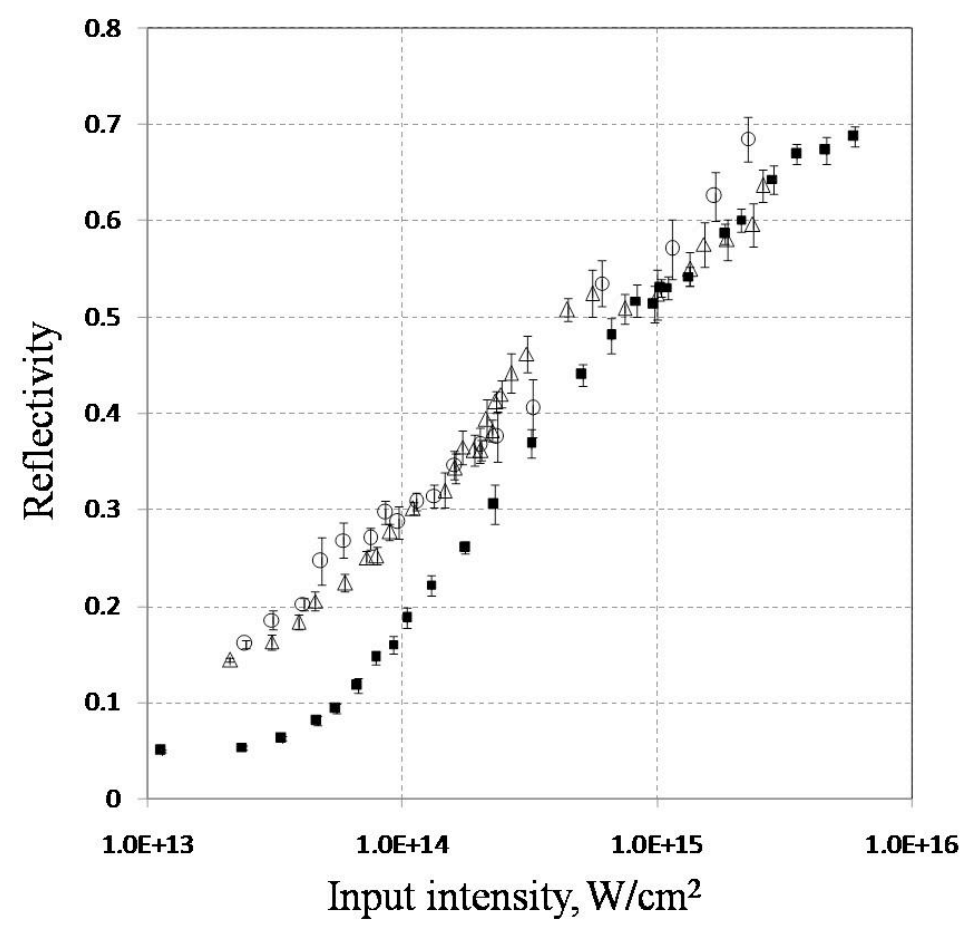

Figure .3. Reflectivity of the water jet at a pressure of 50 Torr (solid squares) and nitrocellulose foil at pressures of 40 Torr (empty triangles) and 10torr (empty circles).

The reflectivity of the plasma mirror as a function of the input pulse intensity is shown in Fig.3. At low power, the observed reflectivity was $5 \%$ in good agreement with the calculated Fresnel reflection value of $6 \%$ using the refractive index of water, $n=1.33$. The plasma mirror 'triggers' around 3-4 x $10^{13} \mathrm{~W} / \mathrm{cm}^{2}$, consistent with the results obtained by other groups using solid targets [13]. The reflectivity curve starts to saturate at $1 \times 10^{15} \mathrm{~W} / \mathrm{cm}^{2}$ reaching maximum reflectivity of $68 \%$ at the input intensity of $5 \times 10^{15}$ $\mathrm{W} / \mathrm{cm}^{2}$. This maximum reflectivity is similar to, but somewhat lower than that demonstrated previously. For example, the authors of [12] observed a maximum reflectivity from a solid target of $80 \%$ at an incidence angle of $6^{\circ}$, however the 
reflectivity for $45^{\circ}$ incidence was only $65 \%$. Similarly the highest reflectivity achieved from an ethylene glycol liquid jet plasma mirror at $45^{\circ}$ was close to $80 \%$ [5].

The pressure in the chamber in our experiments was around 50 Torr - significantly higher than used in solid target or ethylene glycol experiments due to the high saturation pressure of water vapor ( $\sim 20$ torr). A spectral analysis of the pulses indicates the presence of a slight amount of blue-shifting after reflection, which is attributed to the result of ionizing the background gas inside the chamber. [14]. Ionization-induced defocusing may therefore play a role in the saturation of the mirror reflectivity at high intensities.

In order to test the influence of the residual gas and compare the performance of the water jet plasma mirror to the one based on a solid target, the reflectivity of a nitrocellulose foil was evaluated. Within the error margin of the measurements, the reflectivity of the foil was the same at a similar pressure in the chamber. Under a reduced pressure of 10 Torr the reflectivity saturated somewhat slower, indicating that the residual gas contributes to the saturation. Beyond this contribution the saturation of the reflectivity at high intensities can be explained by laser absorption in plasma. Doumy et. al.[15] simulated the response of a silica plasma mirror using one dimensional Lagrangian fluid code. In this simulation, saturation of the reflectivity at $65 \%$ was observed due to collisional absorption in plasma. Their model predicted a further increase of the reflectivity above a plateau at cumulative fluences above $100 \mathrm{~J} / \mathrm{cm}^{2}$ due to the heating of the plasma and decrease in the collision rate. Although a maximum fluence in our experiments was of the order of $2 * 10^{3} \mathrm{~J} / \mathrm{cm}^{2}$, the predicted increase in reflectivity has not yet been observed. One possible explanation for this discrepancy may be that a lower plasma density is obtained from water compared to silica-based targets due to the fact that the silicon atoms are capable of contributing more electrons per atom than hydrogen. Further numerical simulation will be required in order to check this conclusion as well as to investigate the possibility of obtaining higher reflectivities. For example, a slight rotation of the laser polarization may result in more efficient heating of the plasma through resonant absorption of the p-polarized component resulting in a decrease in collisional absorption and increase of the overall reflectivity.

In order to check the quality of the spatial mode, the reflected beam was refocused by a conjugate lens pair and imaged onto a CCD with a 10-x microscope objective. In order to benchmark the reflected spots against the quality of the original laser mode, the water jet surface was replaced with a BK-7 wedge. The wedge was positioned slightly upstream off the focus of the input laser beam in order to avoid the glass breakdown.

The lineouts of the images of the input beam and of the beam reflected from the water jet surface are shown in Figure 4. The water jet reflection was recorded at a laser intensity below the plasma mirror onset threshold and at a laser power corresponding to the maximum reflectivity of the plasma mirror. The beam at the full power has a top- 

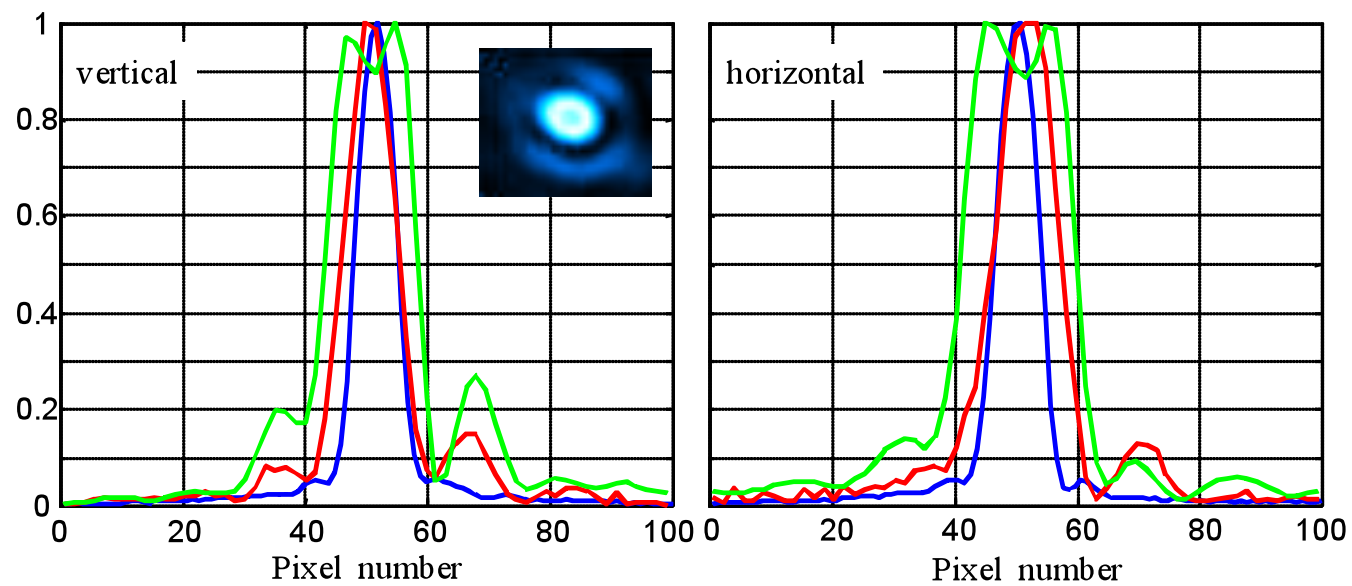

Figure 4. Vertical (left) and horizontal (right) line-outs of the spatial mode profiles refocused with a conjugate pair. Blue curve shows profile of the beam when reflected off a glass window positioned slightly upstream of the focus, as a reference Red curve shows the beam reflected off the water jet at the intensity below the optical breakdown. Green curve is a spot at maximum reflectivity. The inset shows a snapshot of the reflected spot at maximum reflectivity.

hat profile, which is consistent with operating at the saturated part of the reflection curve in Fig. 3. The beam reflected off the water jet, both at low and high laser intensities, appears astigmatic compared to the beam at the absence of the water jet. This effect can stem from the slight curvature of the water jet surface, which will produce astigmatism at a reflection angle of $45^{\circ}$. Although interference measurements in air (see Fig.1.) did not indicate significant curvature of the jet, the curvature could increase at vacuum conditions due to increased pressure differential between the liquid and the surrounding gas. Unfortunately the geometry of the vacuum chamber did not allow interferometric measurements of the curvature, which will be the subject of the future work.

\section{CONCLUSIONS}

In conclusion, the feasibility of producing a plasma mirror based on a flowing water film has been demonstrated. The plasma mirror shows nearly $70 \%$ reflectivity which is comparable to results demonstrated with solid-state targets, while having the advantage of automatic renewal of the surface which may become a critical feature with the development of higher repetition rate multi-TW laser systems. Additionally, using water as a target eliminates the problem of carbon-containing debris.

It should be noted that incorporating a water-based plasma mirror into an ultrafast laser beam line requires locally a relatively high pressure zone, with pressure higher than the saturated vapor pressure of water ( $\sim 20$ Torr at room temperature). As this pressure is several orders of magnitude higher than pressures typically used in vacuum chambers for laser-plasma interaction physics and hence requires a differential pumping system is required in order to maintain acceptable pressure in the beam line. Such systems have been developed previously for round capillary water jets used as targets for x-ray generation [16]. 
The authors would like to acknowledge R. Mathies and C. Stewart of UC Berkeley for their advice during the initial design of the wire guided jet, O. Albert of LOA, France for the development of the XPW system, D. Syversrud and N. Ybarrolaza for technical support. This work is supported by the Director, Office of Science, High Energy Physics, US DOE, under Contract no. DE-AC02-05CH11231.

\section{REFERENCES}

[1] H.C. Kapteyn, M.M. Murnane, A. Szoke, and R.W. Falcone, "Prepulse energy suppression for high-energy ultrashort pulses using self-induced plasma shuttering," Optics Letters, vol. 16, Apr. 1991, pp. 490-492.

[2] B. Dromey, S. Kar, M. Zepf, and P. Foster, "The plasma mirror-A subpicosecond optical switch for ultrahigh power lasers," Review of Scientific Instruments, vol. 75, 2004, p. 645.

[3] C. Thaury, F. Quéré, J.P. Geindre, A. Levy, T. Ceccotti, P. Monot, M. Bougeard, and F. Réau, "Plasma mirrors for ultrahigh-intensity optics," Nature Physics, vol. 3, 2007, pp. 424-429.

[4] W. Leemans and E. Esarey, "Laser-driven plasma-wave electron accelerators," Physics Today, vol. 62, 2009, p. 44.

[5] S. Backus, C.G. Durfee III, M.M. Murnane, and H.C. Kapteyn, "High power ultrafast lasers," Review of Scientific Instruments, vol. 69, Mar. 1998, pp. 12071223.

[6] R. Bobkowski and R. Fedosejevs, "Particle emission debris from a KrF laserplasma x-ray source," Journal of Vacuum Science \& Technology A: Vacuum, Surfaces, and Films, vol. 14, 1996, p. 1973.

[7] S. Backus, H.C. Kapteyn, M.M. Murnane, D.M. Gold, H. Nathel, and W. White, "Prepulse suppression for high-energy ultrashort pulses using self-induced plasma shuttering from a fluid target," Optics Letters, vol. 18, Jan. 1993, pp. 134-136.

[8] M.J. Tauber, R.A. Mathies, X. Chen, and S.E. Bradforth, "Flowing liquid sample jet for resonance Raman and ultrafast optical spectroscopy," Review of Scientific Instruments, vol. 74, Nov. 2003, pp. 4958-4960.

[9] T.B. Benjamin, "Wave Formation in Laminar Flow down an Inclined Plane," Journal of Fluid Mechanics, vol. 2, 1957, pp. 554-573.

[10] H.J. Palmer, "The hydrodynamic stability of rapidly evaporating liquids at reduced pressure," Journal of Fluid Mechanics Digital Archive, vol. 75, 2006, pp. 487-511.

[11] P. Manneville, "Rayleigh-Benard Convection: Thirty Years of Experimental, Theoretical, and Modeling Work," Springer Tracts in Modern Physics, vol. 207, 2006, p. 41.

[12] A. Jullien, O. Albert, F. Burgy, G. Hamoniaux, J.P. Rousseau, J.P. Chambaret, F. Augé-Rochereau, G. Chériaux, J. Etchepare, N. Minkovski, and others, "10^-10 temporal contrast for femtosecond ultraintense lasers by cross-polarized wave generation," Optics letters, vol. 30, 2005, pp. 920-922.

[13] C. Ziener, P.S. Foster, E.J. Divall, C.J. Hooker, M.H.R. Hutchinson, A.J. Langley, and D. Neely, "Specular reflectivity of plasma mirrors as a function of intensity, 
pulse duration, and angle of incidence," Journal of Applied Physics, vol. 93, 2003, p. 768.

[14] E. Esarey, G. Joyce, and P. Sprangle, "Frequency up-shifting of laser pulses by copropagating ionization fronts," Physical Review A, vol. 44, 1991, p. 3908.

[15] G. Doumy, F. Qur, O. Gobert, M. Perdrix, P. Martin, P. Audebert, J.C. Gauthier, J. Geindre, and T. Wittmann, "Complete characterization of a plasma mirror for the production of high-contrast ultraintense laser pulses," Physical Review E, vol. 69, Feb. 2004, p. 026402.

[16] U. Vogt, H. Stiel, I. Will, P.V. Nickles, W. Sandner, M. Wieland, and T. Wilhein, "Influence of laser intensity and pulse duration on the extreme ultraviolet yield from a water jet target laser plasma," Applied Physics Letters, vol. 79, 2001, p. 2336. 


\section{DISCLAIMER:}

This document was prepared as an account of work sponsored by the United States Government. While this document is believed to contain correct information, neither the United States Government nor any agency thereof, nor The Regents of the University of California, nor any of their employees, makes any warranty, express or implied, or assumes any legal responsibility for the accuracy, completeness, or usefulness of any information, apparatus, product, or process disclosed, or represents that its use would not infringe privately owned rights. Reference herein to any speci_c commercial product, process, or service by its trade name, trademark, manufacturer, or otherwise, does not necessarily constitute or imply its endorsement, recommendation, or favoring by the United States Government or any agency thereof, or The Regents of the University of California. The views and opinions of authors expressed herein do not necessarily state or re ect those of the United States Government or any agency thereof or The Regents of the University of California. 